\title{
Molecular kinetics of proteins at the surface of porcine sperm before and during fertilization
}

\author{
P.S. Tsai and B.M. Gadella \\ Department of Biochemistry and Cell Biology, Department of Farm Animal Health, Faculty of \\ Veterinary Medicine, Utrecht University, Yalelaan 2, 3584 CM Utrecht, The Netherlands
}

\begin{abstract}
Fertilization is a decisive moment in life and enables the combination of the DNA from two gametes to ultimately form a new organism. The sperm surface, especially the head area, has distinguishable subdomains that are involved in distinct fertilization processes. It is known that the sperm head surface undergoes constant remodelling during epididymal maturation and migration in the male and female genital tract. But intriguingly, the identity, origin and spatial ordering of proteins at the sperm surface that are involved in mammalian fertilization are essentially unknown. This review deals with sperm surface protein modifications that are under somatic cell control. As soon as the sperm is released from the seminiferous tubules it is subjected to these modifications. These surface reorganisations continue until the sperm reside in the fallopian tube where they meet the oocyte and may fertilize it. Most likely, a selective process allows only functionally mature and intact sperm to optimally interact and fertilize the oocyte. Recent data suggest that even the perivitelline fluid is involved in sperm surface remodelling as it contains factors which could facilitate the first penetrating sperm to fertilize the oocyte. In this contribution, the kinetics of proteins at the sperm surface will be overviewed. Better understanding of this would help to design strategies to improve male fertility or to devise novel contraceptives.
\end{abstract}

\section{Introduction}

Although it is still not clear how sperm fertilize the oocyte, the general consensus is that only functionally mature sperm can fertilizes the oocyte and this is somehow accomplished at the surface of the sperm head (Yanagimachi 1994). The sperm is a highly polarized cell with a minimum of cytosol and organelles (Eddy \& O'Brien 1994). The sperm head consists of the nucleus that houses the male haploid genome which is highly condensed together with protamines in the late haploid phase of spermatogenesis, and a large secretory granule called the acrosome which is oriented over the anterior area of the sperm nucleus. At the distal part of the sperm head the flagellum sprouts. In the mid-piece of this flagellum mitochondria are spiralled around the microtubules of the flagellum. In the tail part, specific cytoskeletal elements surround the microtubules of the flagellum. The surfaces of the sperm head, mid-piece and the tail parts of the sperm are heterogeneous (Phelps et al. 1988, Gadella et al. 1995) and reflect the polar distributed organelles that lie under the surface. In particular, the sperm head surface is heterogeneous and at least three subdomains can be distinguished which have separate 
functions in the fertilization process. In general, the sperm has lost many somatic cell features and doesn't house an endoplasmic reticulum, Golgi, lysosomes or peroxisomes. Moreover, the loss of ribosomes disables the sperm's capability to express genes (both transcription as well as translation processes are silenced (Boerke et al. 2007)). Furthermore, due to the removal of almost the entire cytoplasm in the testis (Eddy \& O'Brien 1994) and during epididymal maturation (Dacheux et al. 2005) the sperm has a typical ordering of the remaining organelles and cytoskeletal elements and probably this polar ordering is reflecting into the lateral domains ordering of the sperm's surface (Gadella et al. 2008).

\section{Function of sperm membrane domains at fertilization}

The subdomains of the sperm head area have diversified functions in the series of processes that are involved in fertilization. The apical ridge area of the sperm head specifically recognizes and binds to the zona pellucida (ZP, van Gestel et al. 2007); a larger area of the sperm head surface (the pre-equatorial domain) is involved in the acrosome reaction which results in the release of acrosome components required for ZP-penetration (Yanagimachi 1994, Flesch \& Gadella 2000). The equatorial segment of the sperm head remains intact after the acrosome reaction and is the specific area that recognizes and fuses with the oolemma (the oocyte's plasma membrane) in order to fertilize the oocyte (Vjugina \& Evans 2008). Although the plasma membrane at the mid-piece and tail of sperm are also heterogeneous, the functions of these surface specialisations are not yet understood (Kan \& Pinto-da Silva 1987). It is possible that they are involved in the organisation of optimal sperm motility characteristics. The sperm surface protein organisation is rather complex and, especially in the sperm head, the surface is subjected to constant dynamical changes evoked by the series of changing environments in the male and female genital tract or during sperm handling as is overviewed in the next section.

\section{Sperm surface kinetics}

The domained surface of sperm is already apparent in testicular sperm (Eddy \& O'Brien 1994), however, the molecular dynamics, involved in the establishment of surface specialisation upon spermatogenesis, is largely unknown. Generally, the polar organization of the extracellular matrix components the cytoskeleton and the cell organelles of the sperm are involved in its heterogeneous surface. In mature spermatids the amount of cytosol is minimal and indeed the observed surface domains mirror the organisation of the acrosome, the post-equatorial nucleus the mitochondria and the fibrous sheath, respectively. Moreover, once liberated in the lumen of the seminiferous tubule, the sperm will start its travel through the male and female genital tract and will meet a sequence of different environments. During this voyage, surface remodelling takes place most likely at any site within the two genital tracts. These constant changes start with stabilization of sperm in the male genital tract and is probably accomplished upon epididymal maturation (Gatti et al. 2004, Dacheux et al. 2005) and by re- and decoating events induced by the accessory fluids combined at ejaculation (Gwathmey et al. 2006, Girouard et al. 2008). Beyond surface rearrangements epididymal maturation also results in the removal of cytoplasmic droplets (the cytosolic remnant of the bridges between spermatids) and the acquisition of sperm motility. In the pig, the most important contributions to seminal fluid originate from the seminal vesicles and the bulbo-urethral gland (see Fig. 1). After their deposition in the female genital tract, the reorganization process continues to ensure its further journey in and prepare the sperm surface for its fertilization task. The removal of extracellular glycoprotein coating (release of decapacitation factors) and further remodelling by (cervical) 
uterine and oviduct secretions activate the sperm to meet the oocyte (in vivo capacitation) (Suarez \& Pacey 2006, Rodriguez-Martinez 2007; see Fig. 2). Surface reorganizations are also induced by the interaction of sperm with cumulus cells and remaining follicular fluid components that surround and impregnate the ZP (Getpook \& Wirotkarun 2007, Gil et al. 2008) as well as in the peri-vitelline space (i.e. the fluid filled space between the ZP and the oolemma) (BarraudLange et al. 2007a,b). All these changing environments may cause surface remodelling of the sperm and thus may influence its potential to fertilize the oocyte.

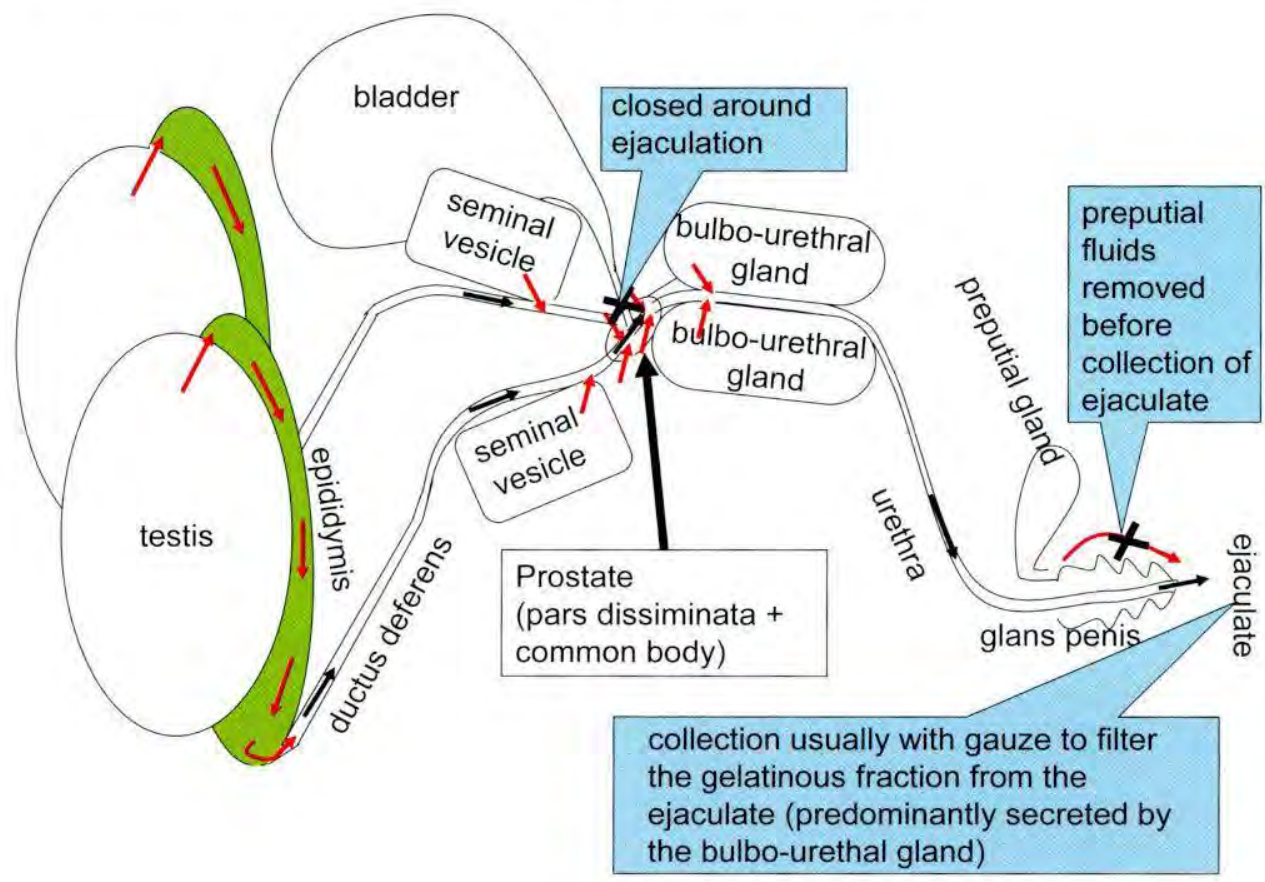

Fig. 1 An overview of the boar reproductive organs. Sperm that leave the testis mature in the epididymis then migrate via the ductus deferens and are mixed with secretions of the indicated accessory sex glands before entering the urethra. Red arrows indicate where the secretions are added to the migrating sperm. The transport of sperm is indicated with black arrows. The epididymis is indicated in green as this is the site where sperm are interacting with epithelia.

The possible mechanisms whereby the sperm surface is altered were reviewed earlier (Gadella 2008) and are summarized in Fig 3. It is very difficult to study sperm surface alterations in situ. However, for many mammalian species, including human, specific sperm handling and incubation media have been optimised for efficient in vitro fertilization purposes. In general, mammalian sperm are activated in a medium that compares with the oviduct in that it contains several capacitation factors, such as high concentrations of bicarbonate, free calcium ions and lipoproteins such as albumin (Flesch \& Gadella 2000). In some species, specific glycoconjugates (Mahmoud \& Parrish 1996) or phosphodiesterase inhibitors are added for extra sperm activation (Barkay et al. 1984). All strategies are designed to evoke capacitation in vitro. This implies that the researcher can observe the relevant sperm surface reorganisation primed under in vitro conditions for fertilization. The membrane composition as well as the ordering of membrane components can be compared with control conditions (media without capacitation factors) or with the membrane ordering of sperm at collection time. Sperm can be 


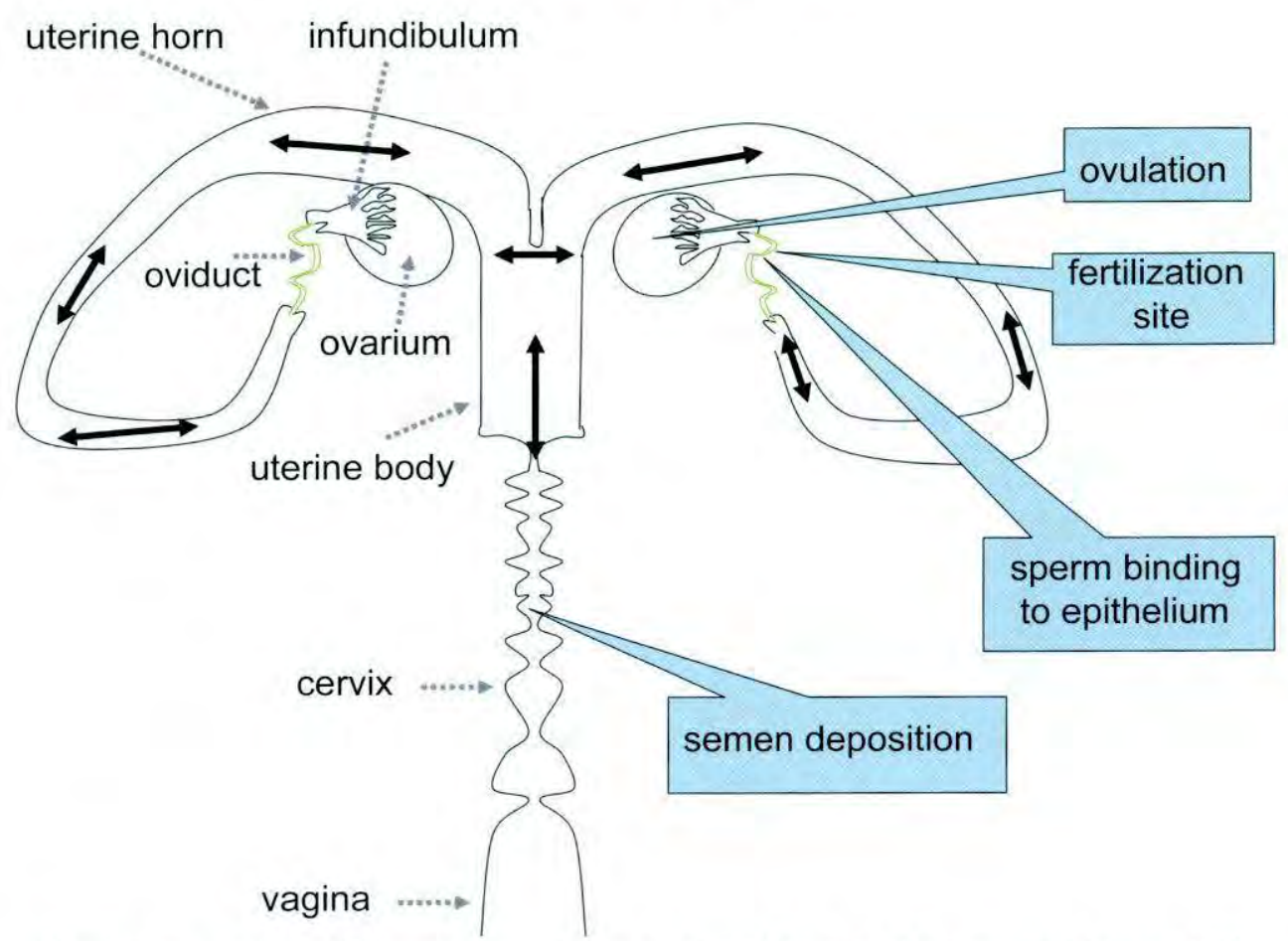

Fig. 2 An overview of the sow reproductive organs. The area where sperm interact with the epithelia is indicated in green (oviduct). Note that the entire female genital tract has been described to be involved in secretory activity. The quantity and composition of the epithelial secretions vary at different sites of the female genital tract and are influenced by the stage of the ovulatory cycle of the sow.

collected from boars where the preputial fluid is removed before collection and the gelatinous fraction is removed by filtering through gauze (see Fig. 1). The collected sperm are washed through a discontinuous density gradient to remove aberrant sperm and non-sperm particles, seminal plasma and factors delaying sperm capacitation (Harrison et al. 1996). The pelletted cells have, after being resuspended into in vitro capacitation media, been extensively studied for the surface reordering of membrane proteins and lipids in the sperm head (for reviews see Flesch \& Gadella 2000, Gadella \& Visconti 2006). Most relevant for fertilization is that sperm surface proteins that are entrapped into small lipid ordered domains (lipid rafts) are clustered into the area that is specifically involved in sperm zona binding (van Gestel et al. 2005) as well as in the docking of the sperm plasma membrane to the outer acrosomal membrane. It is necessary to stress the importance of the sperm surface reordering and changes in composition of membrane components by diverse extracellular factors. The induced lateral redistribution of membrane components appears to also be instrumental for the assembly of a functional sperm protein complex involved in sperm-zona binding as well as for the zona-induction of the acrosome reaction (Fig. 4; Ackermann et al. 2008, Tsai et al. 2007, van Gestel et al. 2007). Therefore, beyond the composition of sperm surface proteins, one needs to study how these proteins are organized and whether they are functionally complex for their physiological role in fertilization. Moreover, the relatively simply defined in vitro capacitation methods probably do not provide all information about sperm surface reorganisation in utero or in the oviduct where hormones and other bioactive non-protein components probably regulate sperm physiology 


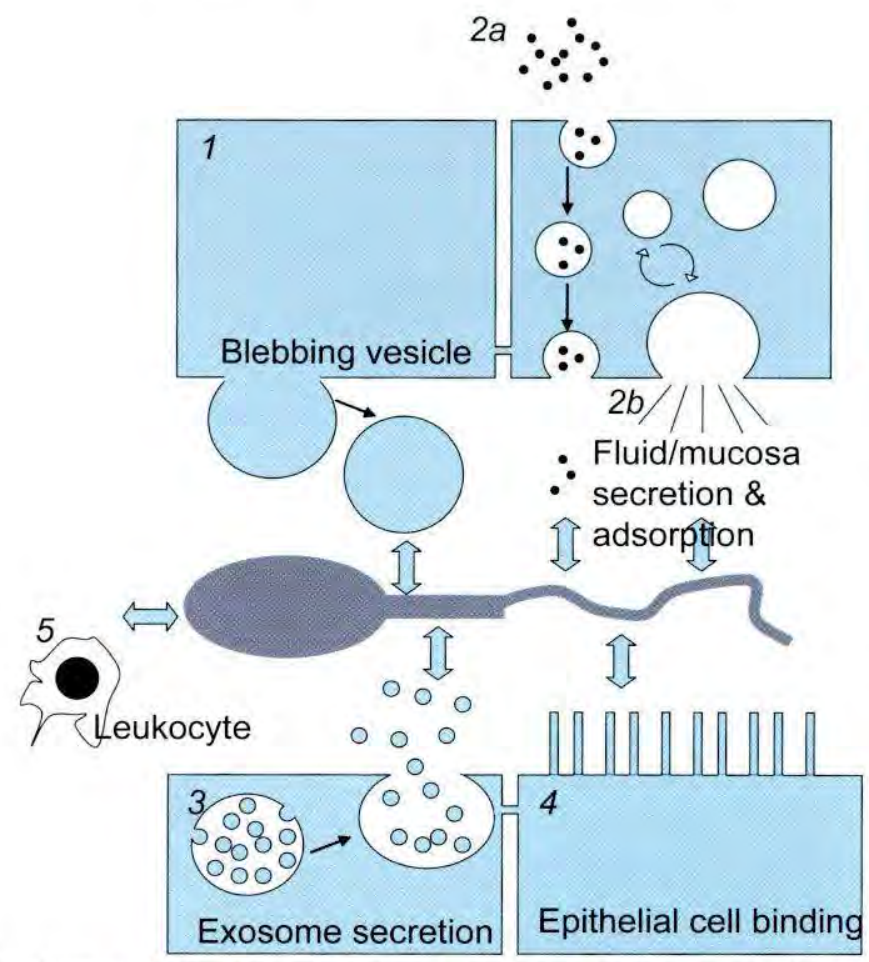

Fig. 3 Hypothetical scheme of possible interactions of male and female genital tract components with the sperm surface. 1. From the diverse epithelia of the male and female genital tract, blebbing vesicles containing cytosol might be released into the genital fluids. Such vesicles may interact and exchange surface components with sperm. It is highly unlikely that such vesicles fuse with the sperm as this would dramatically increase the volume of sperm (which has been reduced maximally in order to obtain an ergonomically designed cell optimally suited for fertilization). Blebbing of vesicles has been demonstrated in the ductus epididymides and the epithelia of the vesicular gland and the prostate of the boar (RodriguezMartinez personal communication). The eventual formation of bleb vesicles in uterus and oviductal epithelia is probably an artefact induced by removing these parts from the pig. 2a. Serum components can be released into the genital fluids by transcytosis (Cooper et al. 1988). Interestingly, lipoprotein particles may invade the surroundings of sperm and may facilitate exchange of larger particles and the sperm surface. $2 \mathrm{~b}$. Fluid phase secretion and adsorption of either fluid or mucosa may directly alter the ECM of sperm. 3. Apocrine secretion of exosomes has been suggested to alter the sperm surface and sperm functioning. Exosomes have been demonstrated to be secreted by the epididymis (epididymosomes) by the prostate (prostasomes) and the uterus (uterosomes) (Gatti et al. 2005, Thimon et al. 2008, Griffiths et al. 2008). Interestingly, exosomes may provide sperm with tetraspanins, a group of membrane proteins involved in tethering of proteins into protein complexes. Recently the addition of CD9 onto the sperm surface by membrane particles has been described to occur even when sperm reaches the perivitellin space (Barraud-Lange et al. 2007 a,b). 4. Sperm interacts with ciliated epithelial cells this has been observed in the epididymis and the oviduct (Gatti et al. 2004, Sostaric et al. 2008), and probably has a physiological role during in situ capacitation. Sperm interactions with other ciliated epithelial cells of the female and male genital tract have not been studied extensively. It is possible that such interactions are important for sperm surface remodelling and for sperm physiology. 5. Semen entering the uterus evokes immunological responses (Schuberth et al. 2008) such as the migration of leukocytes into the uterine fluid (Taylor et al. 2008) which may affect the surface of sperm but most likely is involved in cleaning the uterine fluid from deteriorated sperm (Woelders \& Matthijs 2001). Probably the sperm fraction involved in fertilization occupies the lower parts of the oviduct (where no leukocyte infiltration takes place) at an earlier occasion than required for the responses in the uterus (Rodriguez-Martinez et al. 2005). 

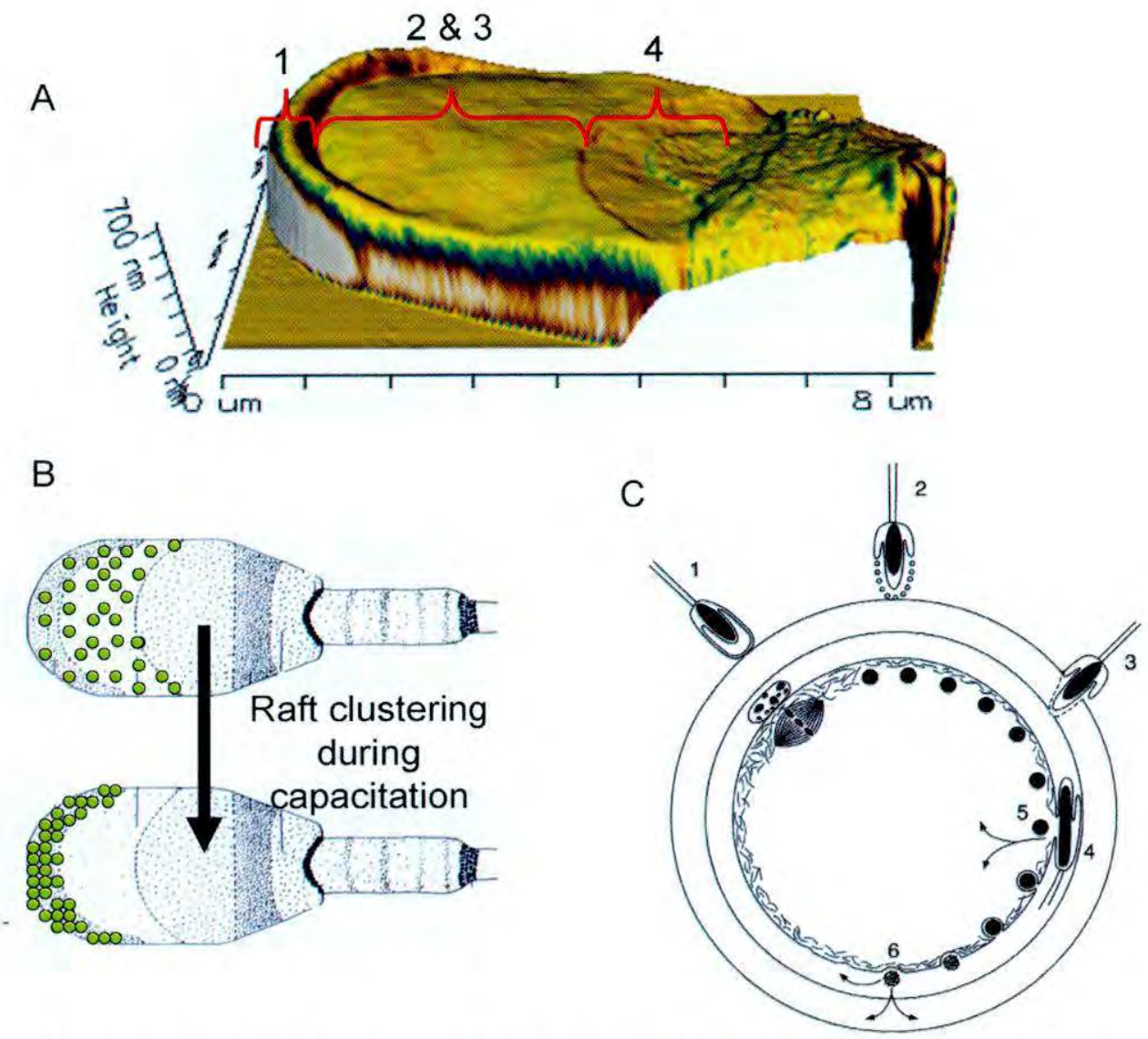

Fig. 4 Protein kinetics at the sperm surface. A. An atomic force microscopic surface view of a porcine sperm head. B. Lipid ordered microdomains at the sperm surface cluster into the apical ridge area of the porcine sperm during in vitro capacitation. $C$. The interactions that the sperm undertakes with the zona pellucida and the oocyte leading to fertilization. 1. zona binding, 2. the acrosome reaction, 3. zona drilling, 4. oolemma binding and fertilization, 5. activation of pronucleus formation and oocyte activation, 6 . induction of a blockade for polyspermic fertilization. The numbers indicated in panel A refer to the specific sperm surface area where these interactions do take place.

differently. Nevertheless we have identified a number of proteins involved in zona binding and in sperm plasma membrane docking with the acrosome using in vitro capacitated sperm (see Fig. 5)

\section{Origin and identification of sperm proteins involved in the cascade leading to in vitro fertilization}

Testicular sperm, just released from the Sertoli cells into the lumen of the seminiferous tubules, are equipped with a number of proteins reportedly involved in ZP-binding. At its surface, the sperm has transmembrane proteins belonging to the ADAMs family, initially thought to be involved in the fertilization process and now reported to be involved in sperm-ZP binding. 


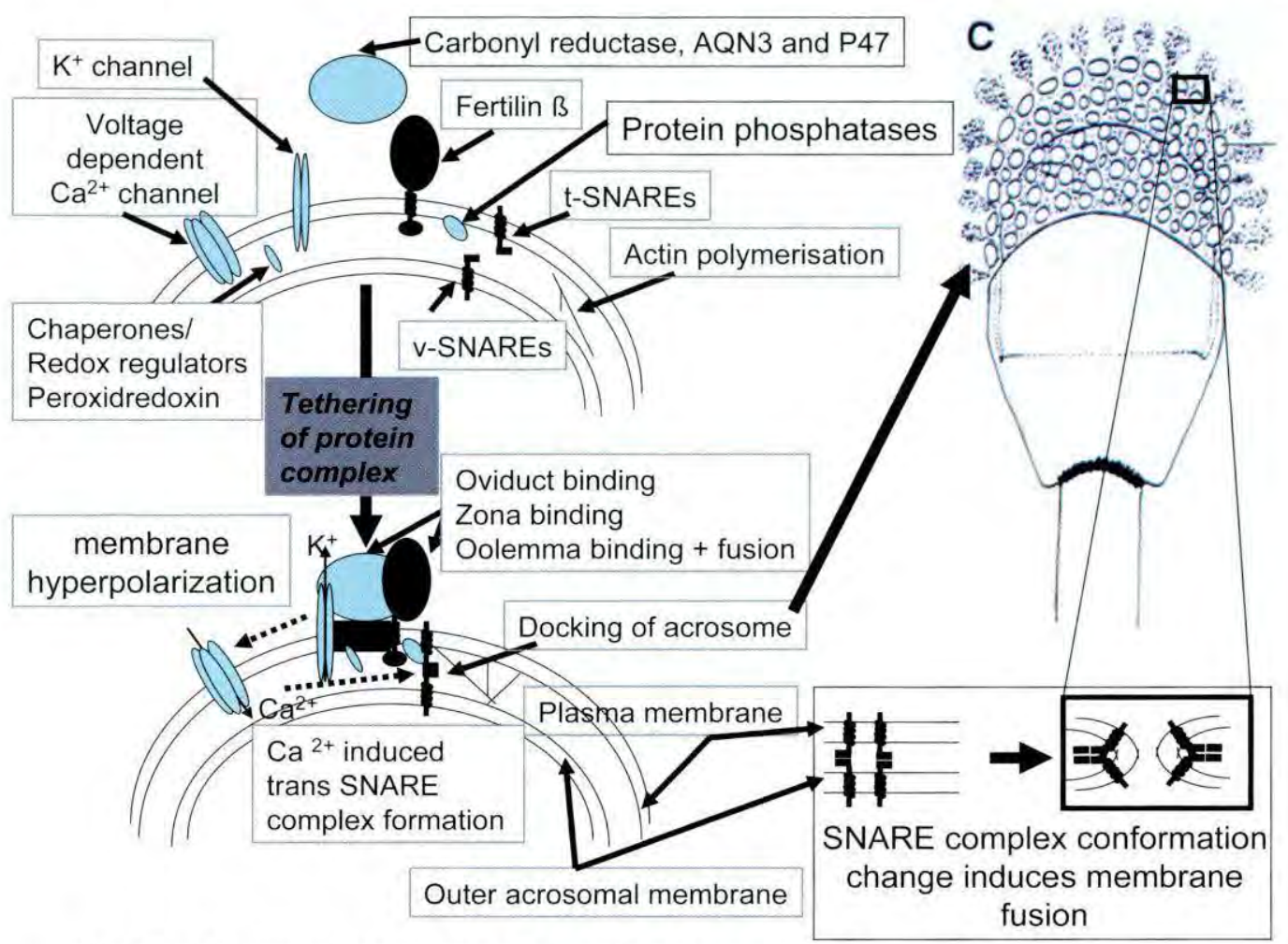

Fig. 5 Left: Summarizing scheme of a hypothetical sperm zona pellucida-binding complex formed during sperm capacitation by raft-induced protein clustering. This may result in a multifunctional protein complex known to play a role in diverse processes leading to fertilization. For explanation and identification of proteins see text. Note that this scheme is based on biochemical and proteomic approaches from epididymal and ejaculated sperm before and after IVF incubations. It is not clear how this picture compares to in situ fertilization where the sperm surface may have been functionally remodelled along the female genital tract. Upper right: Multiple membrane fusions involved in the acrosome reaction exclusive for the anterior part of the sperm head surface. Lower right: Molecular impression of trans SNARE fusion complexes that show a $\mathrm{Ca}^{2+}$ dependent configuration changes required for these membrane fusions (from Tsai \& Gadella, unpublished observations).

ADAM-2, also named fertilin $\beta$, has such a function on boar sperm (van Gestel et al. 2007). Other testicular sperm proteins such as sperm lysosomal like protein (SLLP1, Herrero et al. 2005), and sperm acrosomal membrane proteins (SAMP14 and 32, Vjugina \& Evans 2008) and Sp56 (Buffone et al. 2008) are involved in secondary ZP-binding as they are localized in the acrosome and only become exposed to the ZP structure after the induction of the acrosome reaction. Some secretory proteins like define CRISP are also involved in sperm-ZP adhesion, sperm oolemma binding or the fertilization fusion (Cohen et al. 2007, Da Ros et al. 2007, Busso et al. 2007a). Interestingly, CRISP 2 is of testicular origin but CRISP 1 and 4 originate from the epididymis (Busso et al. 2007b). The exact way in which CRISPs are associated to the sperm surface is not yet known, although CRISP 1 is one of the abundant proteins in epididymosomes (Thimon et al. 2008). Epididymosomes are also reported to influence the lipid composition of the sperm surface (Rejraji et al. 2006). Other proteins that have been shown to be added 
to the sperm surface in the epididymis are P47 or SED1, known to have a role in oviduct and ZP-binding (Shur et al. 2006). In porcine, the spermadhesin AQN-3 and carbonyl reductase are also added to the sperm surface (Ekhlasi-Hundrieser_et al. 2002). Inhibition of hamster carbonyl reductase activity caused a decreased affinity for the ZP while the sperm remained motile and intact (Montfort et al. 2002). Even under very stringent detergent conditions AQN-3 was still able to bind to the ZP (van Gestel et al. 2007). Proteomic analyses identified proteins that do not have a function directly in sperm ZP-binding but that are associated into a ZP-binding protein complex (van Gestel et al. 2007). Some of these proteins are involved in sperm signalling (such as protein phosphatases), while others are involved in the redox balance (peroxiredoxin 5). The latter include a potassium channel which might induce membrane hyperpolarization by $\mathrm{K}^{+}$efflux. This hyperpolarization may in turn open voltage dependent $\mathrm{Ca}^{2+}$ channels that enable $\mathrm{Ca}^{2+}$ dependent processes in the capacitating sperm. An interesting observation was that the major ZP-binding proteins listed above tend to aggregate in capacitating sperm (under IVF conditions) at the surface area involved in ZP-binding i.e. the apical head area (van Gestel et al. 2005,2007 ) and that this coincided with the attraction of SNAREs (soluble N-ethylmaleimidesensitive factor attachment protein receptor) involved in the acrosome reaction (Tsai et al. 2007). The fact that both outer acrosomal SNAREs and plasma membrane SNAREs were observed after capacitation led to the assumption that the lipid ordered membrane aggregation is a preparative step for the acrosome reaction (as proposed in Fig 4). The identified ZP-interacting protein complex is thus not only involved in sperm-ZP binding but may link this event with preparative steps for the acrosome reaction.

In our approach, we only identified the major ZP-binding proteins of the sperm plasma membrane but did not consider the identification of minor proteins or hidden proteins (covered on 2 D IEF-SDS-PAGE gels by the glycosylated ZP-proteins). Therefore, we cannot exclude that we have missed sperm surface proteins involved in sperm-ZP interactions. Although we did not find any proteins originating from one of the accessory male sex glands (Fig. 1) it cannot be excluded that these glands also play a role in sperm surface modification during ejaculation. For instance, the possibility that prostasomes could fuse with sperm (Burden et al. 2006) is interesting but needs to be experimentally validated. In any case, no prostate-derived proteins have this far been described to be involved in sperm-ZP interaction although these fluids are known to have an influence on the sperm surface organisation and protein composition (Russell et al. 1984).

\section{Surface modifications at the female genital tract}

As mentioned above, far less is known about the contribution of the female genital tract to the ZP-binding processes in mammals (Fig. 2). Sperm reside for hours to days in the cervix, uterus and eventually the isthmic part of the oviduct (depending on species for time and deposition place). In pigs, the semen is normally deposited in the cervix. Although there are no data on the role of cervix epithelia and secretory products, it is well established that small numbers of sperm can be inseminated when deposition is directly in the uterine body (Behan \& Watson 2006, Roberts \& Bilkei 2005). Moreover, a very low dose of sperm can be used for deep intrauterine insemination (for review see Vazquez et al. 2005, 2008). A recent ex vivo study using a lectin competition binding assay in uterine segments established that binding of sperm to the uterine epithelium was carbohydrate-dependent (Taylor et al. 2008). However, the proteins involved in this binding are not identified and it is also not clear whether this binding is selective, thus allows only small numbers of sperm to migrate deeper in the uterus or that whether this binding allows modification of the sperm surface that is needed in later processes 
leading to fertilization. The composition of uterine fluid and its effects on the sperm surface or sperm functioning has been completely neglected by most researchers. The only study on the effects of porcine uterine fluids on sperm reports on lipid modifications in sperm membranes (Evans et al. 1987). Porcine sperm contain non-genomic progesterone receptors at the plasma membrane (Jang \& Li 2005) and most likely this hormone binding at the sperm surface is part of the in vivo sperm capacitation process. Murine uterine fluids have been shown to contain exosomes. Interestingly these particles called uterosomes contain the sperm adhesion molecule SPAM-1 and other GPI-linked proteins that can be exchanged with cauda epididymal sperm (Griffiths et al. 2008). It is possible that this exchange improves the sperm's capacity to fertilize the oocyte. However, this possibility is not yet established nor is the presence of uterosomes in the pig species. When semen enters the uterus, it elicits immunological responses which can be observed by a migration of leukocytes (predominantly polymorphonuclear neutrophils; PMNs) into the uterine fluid (Lovell \& Getty 1960, Schuberth et al. 2008, Taylor et al. 2008). It is not clear whether this infiltration will affect sperm that later enter the oviduct but leukocytes clearly reduce the amount of sperm that will enter the oviduct by phagocytosis (Woelders \& Matthijs 2001). It is also uncertain whether phagocytosis is selective (for aberrant sperm) or only unselectively depletes the amount of sperm that migrate further to the oviduct, which is free of leukocytes and the site where sperm are capacitated in vivo in order to fertilize the oocyte (Suarez 2008). In pigs the role of leukocyte infiltration on fertilization is questionable as the vanguard cohort of sperm that occupy the oviduct have been shown to reside and bind to the oviduct epithelia of the isthmic region within 30 minutes after insemination (RodriguezMartinez et al. 2005) which is long before the invasion of PMNs into the uterus.

A number of reports have shown that fluids from the oviduct stimulate sperm capacitation and induce hyperactivated sperm motility. One of the factors involved in this sperm activation is bicarbonate, which is also commonly used for IVF treatments (Rodriguez-Martinez 2007). Oviduct-specific glycoproteins (OSG) as well as osteopontin have been shown to support fertilization in the cow and are secreted by the oviduct (Killian 2004). A sperm binding glycoprotein from the oviductal fluid has recently been shown to induce porcine sperm capacitation (Teijeiro et al. 2008). The lower part of the oviduct is considered to function as the sperm activation site, making sperm capable to meet and fertilize the oocyte. In the isthmus, small numbers of sperm are bound and become capacitated in vivo, there, sperm await to be released at ovulation, to migrate to the upper part of the oviduct (the ampulla) and to fertilize the passing oocyte(s) (for review see Suarez 2008). To this end, the oviduct epithelia and fluids contain sperm binding factors as well as sperm releasing factors that are causing sperm adhesion and release in the correct timing around ovulation (for binding and release characteristics in the bovine oviduct see also Sostaric et al. 2008). Most likely, spermadhesins such as AQN-1 are involved in the formation of the oviductal sperm reservoir as they are involved in sperm binding to this specific epithelium (Ekhlasi-Hundrieser et al. 2005). Note that some spermadhesins (DQH, Manaskova et al. 2007, AQN-3, AWN, P47, Gadella 2009) are added to the sperm phase during epididymal sperm maturation, others (like PSP-I /PSP-Il subunits) are partialy added in the testis (Garcia et al. 2009). Interestingly, recently oviduct-specific glycoproteins have been shown to modulate sperm-ZP interaction and to control the polyspermic fertilization rates in pigs (Hao et al. 2006, Coy et al. 2008). Polyspermy is a well recognised problem in pig IVF and prevention of this unwanted phenomenon may be accomplished by additions of oviductal fluid components to the IVF media. Oviduct epithelial annexins have been suggested to immobilize bovine sperm (by binding bovine sperm proteins BSP; Ignotz et al. 2007). Annexin A2 has also been proposed to be involved in sperm-oviduct binding in the sow (Teijero et al. 2009). In the bovine species this interaction is reversed by oviductal fluid factors such as catalase (Lapointe \& 
Sirard 1998). Catalases that secreted from oviduct may protect sperm from peroxidation damage as is demonstrated in the cow (Lapointe et al. 1998). The interplay of varying glycoproteins at the surface of sperm and oviduct epithelia or oviductal fluid as well as the varying amounts and composition of glycosidases probably orchestrate proper sperm activation just around ovulation in the pig (Carrasco et al. 2008, Töpfer-Petersen et al. 2008). However, the effect of oviduct and uterine proteins on sperm-ZP binding as well as their putative association to the sperm surface is not yet established. Of course it is possible that secreted products of the female genital tract enhance sperm-ZP binding and that more protein candidates from the female genital tract should be added to the surface of the ZP-interacting sperm. In this respect, also the ZP itself, besides being a binding target, may add proteins to the sperm surface. The cumulus cells and the ZP were impregnated with follicular fluid and remnants of this fluid probably will remain attached to the cumulus oocyte complex. For instance, different growth factors and extracellular matrix components have been involved in interactions of sperm to the cumulus oocyte complex (for review see Einspanier et al. 1999). Sperm that interact with these structures may respond to these fluid components like they do to extracted follicular fluid (hyperactivated motility, Getpook \& Wirotkarun 2007, Gil et al. 2007). It should also be mentioned that the oviductal fluid has also been found to be supportive for early embryonic development of the fertilized pig oocyte (Hao et al. 2008, Lloyd et al. 2009).

Finally, an interesting observation has recently been made showing that membrane remodelling occurs after the acrosome reaction when sperm reach the perivitelline space but before the fertilization fusion. Within the perivitelline space, membrane fragments containing CD9 are added to the sperm surface (Barraud-Lange et al. 2007a,b). If correct, this would demonstrate the "bestowment principle" that may exist in mammalian reproduction as the oocyte facilitates the first incoming sperm in the perivitelline space to fertilize by transferring functional tethering proteins to the surface of sperm cells. It remains to be established whether such process also enables oviductal sperm to bind to the ZP. It may also be mentioned that sperm proteins involved in oolemmal binding and the fertilization fusion are reported for mouse and human sperm (Ellerman et al. 2006, Vjugina \& Evans 2008) but data for boar sperm are scarce.

\section{Conclusions}

The continuous sperm surface remodelling that occurs during sperm transit from the rete testis towards the oviduct and possibly even within the peri-vitelline space and the physiological role of this surface kinetics is, to a large extent, terra incognita. The identification of different complex proteins systems within the male and female genital tract is promising; however, their role and function in events associated with sperm-oocyte interactions are still difficult to test (Boerke et al. 2008).

Wild-type and genotypically knock-out mice have already been used to validate the function of certain proteins proposed to play part in sperm ZP-binding, the acrosome reaction, oolemma binding as well as oolemma-sperm fusion (for an overview see Vjugina \& Evans 2008). Such approaches delivered some valuable information on the potential impact of certain proteins involved in mammalian fertilization. However, the molecular intervention of transcription and translation in gametes is hampered by the fact that in sperm both processes are silenced and in the oocyte almost all mRNA is stored for post-fertilization translation. Therefore, it is possible to intervene with molecular processes involved in either gametogenesis or postfertilization development rather than the molecular processes required for gamete interaction and fertilization. An example of this is a mutation in spermatogenic cells of the syntaxin2/ 
epimorphin gene. The protein translated from this gene plays, according to our research, a role in the acrosome reaction (Tsai et al. 2007) but the mutation causes a defect in the transition from spermatocyte to spermatids. Thus, a phenotypic knock-out of syntaxin 2 cannot be used to study the effect of this protein on fertilization simply because the knock-out phenotype fails to produce sperm (Akiyama et al. 2008). Furthermore, in many cases homologous genetic recombination applications have shown that knocking out the expression of phenotypic factors that were previously believed to be essential for fertilization were found to be dispensable to this process (Okabe \& Cummins 2007). This could be partly explained by the fact that biological systems contain redundancies and compensatory mechanisms and both processes are believed to play a prominent role in the evolution of gamete interaction and thus in speciation (Turner \& Hoekstra 2008, Herlyn \& Zischler 2008). On the other hand, future outcome from genomic approaches devoted to study the molecular mechanisms involved in mammalian fertilization may also indicate that a substantial modification of classical fertilization models is required (Okabe \& Cummins 2007).

The production of knock-out pigs is very expensive and time consuming but it is possible to isolate specific structures of the male or female genital tract from pigs. Gene specific silencing of protein translation is possible with interference RNA technology. In this way the specific role of proteins in fertilization and in sperm surface kinetics can be studied. The big problem is that the treatment of explants and cells cause dedifferentiation and alter their interaction with sperm (Sostaric et al. 2008).

Therefore, after leaving the testis, sperm are subjected to a series of events causing continuous sperm surface remodelling, with specific sperm surface protein kinetics, relevant for its final fertilization task. Difficulties in molecular intervention approaches as well as the difficulties to study sperm surface remodelling in situ are nowadays compensated by high throughput proteomic technologies that allow identification of low abundant proteins. In combination with off-gel full LC-MS/MS platforms, sperm surface isolation and purification technologies, isobaric tagging strategies for peptides (Ernoult et al. 2008, Zieske 2006) will enable us to cover the full sperm surface proteome (Gadella 2009) in the near future, when the full pig genome and its annotation will become accessible to the public (expected early 2010, Churcher, personal communication).

\section{References}

Ackermann F, Zitranski N, Heydecke D, Wilhelm B, Gudermann T \& Boekhoff I 2008 The Multi-PDZ domain protein MUPP1 as a lipid raft-associated scaffolding protein controlling the acrosome reaction in mammalian spermatozoa. Journal of Cellular Physiology 214 757-768.

Akiyama K, Akimaru S, Asano Y, Khalaj M, Kiyosu C, Masoudi AA, Takahashi S, Katayama K, Tsuji T, Noguchi J \& Kunieda T 2008 A new ENU-induced mutant mouse with defective spermatogenesis caused by a nonsense mutation of the syntaxin 2/epimorphin (Stx2/Epim) gene. lournal of Reproduction and Development 54 122-128.

Barkay J, Bartoov B, Ben-Ezra S, Langsam J, Feldman E, Gordon S \& Zuckerman H 1984 The influence of in vitro caffeine treatment on human sperm morphology and fertilizing capacity. Fertility and Sterility $\mathbf{4 1}$ 913-918.

Barraud-Lange V, Naud-Barriant N, Bomsel $M$, Wolf
JP \& Ziyyat A 2007a Transfer of oocyte membrane fragments to fertilizing spermatozoa. FASEB / 21 3446-3449.

Barraud-Lange V, Naud-Barriant N, Saffar L, Gattegno L, Ducot 8, Drillet AS, Bomsel M, Wolf JP \& Ziyyat A 2007 b $\alpha 6 \beta 1$ integrin expressed by sperm is determinant in mouse fertilization. BMC Developmental Biology 7102.

Behan JR \&Watson PF 2006 A field investigation of intracervical insemination with reduced sperm numbers in gilts. Theriogenology 66 338-343.

Boerke A, Dieleman S] \& Gadella BM 2007 A possible role for sperm RNA in early embryo development. Theriogenology 68 147-155.

Boerke A, Tsai PS, Garcia-Gil N, Brewis IA \& Gadella BM 2008 Capacitation-dependent reorganization of microdomains in the apical sperm head plasma membrane: functional relationship with zona binding and the zona-induced acrosome reaction. 
Theriogenology 70 1188-1196.

Buffone MG, Zhuang T, Ord TS, Hui L, Moss SB \& Gerton GL 2008 Recombinant mouse sperm ZP3-binding protein (ZP3R/sp56) forms a high order oligomer that binds eggs and inhibits mouse fertilization in vitro. Journal of Biologial Chemistry 283 12438-12445.

Burden HP, Holmes CH, Persad R \& Whittington $K$ 2006 Prostasomes--their effects on human male reproduction and fertility. Human Reproduction Update 12 283-292.

Busso D, Goldweic NM, Hayashi M, Kasahara M \& Cuasnicú PS 2007a Evidence for the involvement of testicular protein CRISP2 in mouse sperm-egg fusion. Biology of Reproduction 76 701-708.

Busso D, Cohen DI, Maldera JA, Dematteis A \& Cuasnicu PS 2007b A novel function for CRISP1 in rodent fertilization: involvement in sperm-zona pellucida interaction. Biology of Reproduction 77 848-854.

Carrasco LC, Romar R, Avilés M, Gadea J, Coy P 2008 Determination of glycosidase activity in porcine oviductal fluid at the different phases of the estrous cycle. Reproduction 136 833-842.

Cohen DJ, Da Ros VG, Busso D, Ellerman DA, Maldera JA, Goldweic N \& Cuasnicú PS 2007 Participation of epididymal cysteine-rich secretory proteins in spermegg fusion and their potential use for male fertility regulation. Asian fournal of Andrology 9 528-532.

Coy P, Cánovas S, Mondéjar I, Saavedra MD, Romar R, Grullón L, Matás C \& Avilés M 2008 Oviductspecific glycoprotein and heparin modulate spermzona pellucida interaction during fertilization and contribute to the control of polyspermy. Proceedings of the National Academy of Sciences U S A 105 15809-15814.

Cooper TG, Yeung CH \& Bergmann M 1988 Transcytosis in the epididymis studied by local arterial perfusion. Cell Tissue Research 253 631-637.

Dacheux JL., Castella S, Gatti JL \& Dacheux F 2005 Epididymal cell secretory activities and the role of proteins in boar sperm maturation. Theriogenology 63319.341.

Da Ros V, Busso D, Cohen DJ, Maldera J, Goldweic N \& Cuasnicu PS 2007 Molecular mechanisms involved in gamete interaction: evidence for the participation of cysteine-rich secretory proteins (CRISP) in spermegg fusion. Society of Reproduction and Fertility Supplement 65 353-356.

Eddy EM \& O'Brien DA 1994 The spermatozoon. In The Physiology of Reproduction. Eds E Knobil \& JD Neild JD pp 29-78 New York: Raven Press.

Einspanier R, Gabler C, Bieser B, Einspanier A, Berisha B, Kosmann M \& Wollenhaupt K, Schams D 1999 Growth factors and extracellular matrix proteins in interactions of cumulus-oocyte complex, spermatozoa and oviduct. Journal of Reproduction and Fertility Supplement 54 359-365.

Ekhlasi-Hundrieser $M$, Gohr K, Wagner A, Tsolova $M$, Petrunkina A \& Töpfer-Petersen E 2005 Spermadhesin AQN1 is a candidate receptor molecule involved in the formation of the oviductal sperm reservoir in the pig. Biology of Reproduction 73 536-545.

Ekhlasi-Hundrieser M, Sinowatz F, Greiser De Wilke 1, Waberski D \& Töpfer-Petersen E 2002 Expression of spermadhesin genes in porcine male and female reproductive tracts. Molecular Reproduction and Develeopment 61 32-41.

Ellerman DA, Myles DG \& Primakoff P 2006 A role for sperm surface protein disulfide isomerase activity in gamete fusion: evidence for the participation of ERp57. Developmental Cell $10831-837$.

Ernoult E, Gamelin E \& Guette C 2008 Improved proteome coverage by using ITRAQ labelling and peptide OFFGEL fractionation. Proteome Science 627.

Evans RW, Weaver DE \& Clegg ED 1987 Effects of in utero and in vitro incubation on the lipid-bound fatty acids and sterols of porcine spermatozoa. Gamete Research 18 153-162.

Flesch FM \& Gadella BM 2000 Dynamics of the mammalian sperm plasma membrane in the process of fertilization. Biochimica et Biophysica Acta 1469 197-235.

Gadella BM 2009 Sperm surface proteomics. In Immune Infertility Eds W Krause \& E Naz Heidelberg, Springer Verlag (in press)

Gadella BM 2008 The assembly of a zona pellucida binding protein complex in sperm. Reproduction of Domestic Animals 43 Supplement 5 12-19.

Gadella BM, Lopes-Cardozo $M$, van Golde $L M$, Colenbrander B \& Gadella TW Jr. 1995 Glycolipid migration from the apical to the equatorial subdomains of the sperm head plasma membrane precedes the acrosome reaction. Evidence for a primary capacitalion event in boar spermatozoa. Journal of Cell Science 108 935-946.

Gadella BM, Tsai PS, Boerke A \& Brewis IA 2008 Sperm head membrane reorganisation during capacitation. International Journal of Developmental Biology 52 473-480.

Gadella BM \& Visconti PE 2006 Regulation of capacitation. In: The Sperm Cell; Production, Maturation, Fertilization, Regeneration. Eds C de Jonge \& C Barratt pp 134-169 Cambridge: Cambridge University Press

García EM, Calvete IJ, Sanz L, Roca J, Martínez EA \& Vázquez JM 2009 Distinct effects of boar seminal plasma fractions exhibiting different protein profiles on the functionality of highly diluted boar spermatozoa. Reproduction in Domestic Animals 44 200-205.

Gatti JL, Castella S, Dacheux F, Ecroyd H, Metayer S, Thimon V \& Dachuex JL 2004 Post-testicular sperm environment and fertility. Animal Reproduction Science 82-83 321-339.

Gatti JL, Métayer S, Belghazi M, Dacheux F \& Dacheux JL 2005 Identification, proteomic profiling, and origin of ram epididymal fluid exosome-like vesicles. Biology of Reproduction 72 1452-1465.

Getpook C \& Wirotkarun S 2007 Sperm motility stimulation and preservation with various concentrations of follicular fluid. Journal of Assisted Reproductive 
Cenetics 24 425-428.

Gil PI, Guidobaldi HA, Teves ME, Uñats DR, Sanchez R \& Giojalas LC 2008 Chemotactic response of frozenthawed bovine spermatozoa towards follicular fluid. Animal Reproduction Science 108 236-246.

Girouard J, Frenette G \& Sullivan R 2008 Seminal plasma proteins regulate the association of lipids and proteins within detergent-resistant membrane domains of bovine spermatozoa. Biology of Reproduction 78 921-931.

Griffiths GS, Galileo DS, Reese $K$ \& Martin-Deleon PA 2008 Investigating the role of murine epididymosomes and uterosomes in $\mathrm{GPI}$-linked protein transfer to sperm using SPAM1 as a model. Molecular Reproduction and Development 75 1627-1636.

Gwathmey TM, Ignotz GG, Mueller IL, Manjunath P \& Suarez SS 2006 Bovine seminal plasma proteins PDC-109, BSP-A3, and BSP-30-kDa share functional roles in storing sperm in the oviduct. Biology of Reproduction 75 501-507.

Hao Y, Mathialagan N, Walters E, Mao J, Lai L, Becker D, Li W, Critser I \& Prather RS 2006 Osteopontin reduces polyspermy during in vitro fertilization of porcine oocytes. Biology of Reproduction 75 726-733.

Hao Y, Murphy CN, Spate L, Wax D, Zhong Z, Samuel M, Mathialagan N, Schatten H \& Prather RS 2008 Osteopontin improves in vitro development of porcine embryos and decreases apoptosis. Molecular Reproduction and Development 75 291-298.

Harrison RA, Ashworth PJ \& Miller NG 1996 Bicarbonate/ $\mathrm{CO} 2$, an effector of capacitation, induces a rapid and reversible change in the lipid architecture of boar sperm plasma membranes. Molecular Reproduction and Development 45 378-391.

Herlyn H \& Zischler H 2008 The molecular evolution of sperm zonadhesin. International lournal of Developmental Biology 52 781-790.

Herrero MB, Mandal A, Digilio LC, Coonrod SA, Maier B \& Herr JC 2005 Mouse SLLP1, a sperm lysozyme-like protein involved in sperm-egg binding and fertilization. Developmenmtal Biology 284 126-42.

Ignotz GG, Cho MY \& Suarez SS 2007 Annexins are candidate oviductal receptors for bovine sperm surface proteins and thus may serve to hold bovine sperm in the oviductal reservoir. Biology of Reproduction 77 906-913.

Jang S \& Yi LS 2005 Identification of a 71 kDa protein as a putative non-genomic membrane progesterone receptor in boar spermatozoa. Journal of Endocrinology 184 417-25.

Kan FW \& Pinto da Silva P 1987 Molecular demarcation of surface domains as established by label-fracture cytochemistry of boar spermatozoa. Journal of Histochemistry and Cytochemistry 35 1069-1078.

Killian G) 2004 Evidence for the role of oviduct secretions in sperm function, fertilization and embryo development. Animal Reproduction Science 82-83 141-153.

Lapointe S \& Sirard MA 1998 Catalase and oviductal fluid reverse the decreased motility of bovine sperm in culture medium containing specific amino acids. lournal of Andrology 19 31-36.

Lapointe S, Sullivan R \& Sirard MA 1998 Binding of a bovine oviductal fluid catalase to mammalian spermatozoa. Biology of Reproduction 58 747-753.

Lloyd RE, Romar R, Matas C, Gutierrez-Adan A, Holt WV \& Coy P 2009 Effects of oviductal fluid on the development, quality and gene expression of porcine blastocyst produced in vitro. Reproduction (In press).

Lovell JE \& Getty R 1960 Cytochemical reactions of porcine spermatids and spermatozoa. American Journal of Veterinary Research 21 597-609.

Mahmoud Al \& Parrish |J 1996 Oviduct fluid and heparin induce similar surface changes in bovine sperm during capacitation: a flow cytometric study using lectins. Molecular Reproduction and Development 43 554-560.

Manásková P, Peknicová J, Elzeinová F, Tichá M \& Jonáková $V 2007$ Origin, localization and binding abilities of boar DQH sperm surface protein tested by specific monoclonal antibodies. Journal of Reproductive Immunology 74 103-113.

Montfort L, Frenette G \& Sullivan R 2002 Sperm-zona pellucida interaction involves a carbonyl reductase activity in the hamster. Molecular Reproduction and Development 61 113-119.

Okabe M \& Cummins JM 2007 Mechanisms of sperm-egg interactions emerging from gene-manipulated animals. Cellular and Molecular Life Sciences 64 1945-1958.

Phelps BM, Primakoff P, Koppel DE, Low MG \& Myles DG 1988 Restricted lateral diffusion of PH-20, a $\mathrm{PI}$-anchored sperm membrane protein. Science $\mathbf{2 4 0}$ 1780-1782.

Rejraji H, Sion B, Prensier G, Carreras M, Motta C, Frenoux JM, Vericel E, Grizard G, Vernet P \& Drevet IR 2006 Lipid remodeling of murine epididymosomes and spermatozoa during epididymal maturation. Biology of Reproduction 74 1104-1113.

Roberts PK \& Bilkei G 2005 Field experiences on post-cervical artificial insemination in the sow. Reproduction in Domestic Animals 40 489-491.

Rodriguez-Martínez $H$, Saravia $F$, Wallgren $M$, Tienthai P, Johannisson A, Vázquez JM, Martinez E, Roca J, Sanz L, Calvete JI 2005 Boar spermatozoa in the oviduct. Theriogenology 63 514-535.

Rodriguez-Martinez, $\boldsymbol{H} 2007$ Role of the oviduct in sperm capacitation. Theriogenology 68 138-146.

Russell L.D, Peterson RN, Hunt W \& Strack LE 1984 Posttesticular surface modifications and contributions of reproductive tract fluids to the surface polypeptide composition of boar spermatozoa. Biology of Reproduction 30 959-978.

Schuberth HJ, Taylor U, Zerbe H, Waberski D, Hunter R \& Rath D 2008 Immunological responses to semen in the female genital tract. Theriogenology 70 1174-1181.

Shur BD, Rodeheffer C, Ensslin MA, Lyng R \& Raymond A 2006 Identification of novel gamete receptors that mediate sperm adhesion to the egg coat. Molecular 
and Cellular Endocrinology 250 137-148.

Sostaric E, Dieleman Sl, van de Lest $\mathrm{CH}$, Colenbrander B, Vos PL \& Garcia-Gil N, Gadella BM 2008 Sperm binding properties and secretory activity of the bovine oviduct immediately before and after ovulation. Molecular Reproduction and Development 75 60-74.

Suarez SS 2008 Regulation of sperm storage and movement in the mammalian oviduct. International Journal of Developmental Biology 52 455-462.

Suarez SS \& Pacey AA 2006 Sperm transport in the female reproductive tract. Human Reproduction Update 12 23-37.

Taylor U, Rath D, Zerbe H \& Schuberth H] 2008 Interaction of intact porcine spermatozoa with epithelial cells and neutrophilic granulocytes during uterine passage. Reproduction in Domestic Animals 43 166-175.

Teijeiro JM, Ignotz GG \& Marini PE 2009 Annexin A2 is involved in pig (Sus scrofa) sperm-oviduct interaction. Molecular Reproduction and Development 76 334-341.

Teijeiro JM, Cabada MO \& Marini PE 2008 Sperm binding glycoprotein (SBG) produces calcium and bicarbonate dependent alteration of acrosome morphology and protein tyrosine phosphorylation on boar sperm. Journal of Cellular Biochemistry 103 1413-1423.

Thimon V, Frenette G, Saez F, Thabet M \& Sullivan R 2008 Protein composition of human epididymosomes collected during surgical vasectomy reversal: a proteomic and genomic approach. Human Reproduction 23 1698-1707.

Töpfer-Petersen E, Ekhlasi-Hundrieser $M$ \& Tsolova M 2008 Glycobiology of fertilization in the pig. International Journal of Developmental Biology 52 717-736.

Tsai PS, De Vries KJ, De Boer-Brouwer M, Garcia-Gil N, Van Gestel RA, Colenbrander B, Gadella BM \&
Van Haeften T 2007 Syntaxin and VAMP association with lipid rafts depends on cholesterol depletion in capacitating sperm cells. Molecular Membrane Biology 24 313-324.

Turner LM \& Hoekstra HE 2008 Causes and consequences of the evolution of reproductive proteins. International Journal of Developmental Biology 52 769-780.

van Gestel RA, Brewis IA, Ashton PR, Brouwers JF \& Gadella BM 2007 Multiple proteins present in purified porcine sperm apical plasma membranes interact with the zona pellucida of the oocyte. Molecular Human Reproduction 13 445-454.

van Gestel RA, Brewis IA, Ashton PR, Helms JB, Brouwers JF \& Gadella BM 2005 Capacitationdependent concentration of lipid rafts in the apical ridge head area of porcine sperm cells. Molecular Human Reproduction 11 583-590.

Vazquez IM, Martinez EA, Roca J, Gil MA, Parrilla I, Cuello C, Carvajal G, Lucas X \& Vazquez IL 2005 Improving the efficiency of sperm technologies in pigs: the value of deep intrauterine insemination. Theriogenology 63 536-547.

Vazquez JM, Roca J, Gil MA, Cuello C, Parrilla I, Vazquez IL. \& Martínez. EA 2008 New developments in lowdose insemination technology. Theriogenology 70 1216-1224.

Vjugina U \& Evans JP 2008. New insights into the molecular basis of mammalian sperm-eg membrane interactions. Frontiers in Biosciences 13 462-476.

Woelders H \& Matthijs A 2001 Phagocytosis of boar spermatozoa in vitro and in vivo. Reproduction Supplement 58 113-127.

Yanagimachi R 1994 In The Physiology of Reproduction. Eds E Knobil \& JD Neild JD pp 189-317 New York: Raven Press

Zieske LR 2006 A perspective on the use of ITRAQ reagent technology for protein complex and profiling studies. Journal of Experimental Botany 57 1501-1508. 